\title{
Obesity and periodontal disease - An overview
}

\author{
R. Vijayalakshmi ${ }^{1 *}$, Thamaraiselvan ${ }^{2}$, Jaideep Mahendra $^{3}$, Sruthi Srinivasan $^{4}$, Burnice Nalina Kumari $^{5}$ \\ ${ }^{\mathbf{1}}$ Associate Professor, ${ }^{\mathbf{2}}$ Reader, ${ }^{3}$ Professor, ${ }^{\mathbf{4}}$ Postgraduate Student, ${ }^{\mathbf{5}}$ Assistant Professor, Dept. of Periodontics, ${ }^{\mathbf{1}, \mathbf{3}-5}$ Meenakshi Ammal Dental \\ College, Chennai, Tamil Nadu, ${ }^{2}$ Saveetha Dental College \& Hospital, Chennai, Tamil Nadu, India \\ *Corresponding Author: R. Vijayalakshmi \\ Email: rajaramvijayalakshmi@gmail.com
}

\begin{abstract}
Obesity is a condition where the utilization and distribution of nutrients and energy status poses to be a challenge at cell and organism levels. Obesity is often associated with increased lipid and blood glucose levels, which may have deleterious consequences for the host response leading to alterations in $\mathrm{T}$ cells and monocytes/macrophages, as well as an increased cytokine production. Periodontitis, is an inflammatory disease process that results in permanent destruction of the supporting apparatus of the teeth, including the loss of gingival connective tissue, destruction of the periodontal ligament and resorption of alveolar bone. It is associated with increased prevalence of cardiovascular morbidity and mortality. There is a rationale that indicates that the presence of periodontal pockets which can harbor pathogenic microorganisms can evoke a host response and elicit a systemic effect. A potential interaction among obesity, periodontitis, and chronic disease incidence has been reported. This literature review aims at elaborating the possible inter-relationship between obesity and periodontal disease. Articles for this overview were procured from search engines google, google scholar and PubMed.
\end{abstract}

Keywords: Obesity, Periodontitis, Inflammation, Cytokines.

\section{Introduction}

The World Health Organization (WHO) defines overweight and obesity as "abnormal or excessive fat accumulation that presents a risk to health. ${ }^{1}$

Obesity is a condition where the utilization and distribution of nutrients and energy status poses to be a challenge at cell and organism levels. The organism cannot adapt and maintain homeostasis under continuous energy and nutrient exposure, and the consequent emergence of metabolic and oxidative stress leads to inflammatory responses and cell organelle dysfunction. ${ }^{2}$

Obesity is often associated with increased lipid and blood glucose levels, which may have deleterious consequences for the host response leading to alterations in $\mathrm{T}$ cells and monocytes/macrophages, as well as an increased cytokine production. This imbalance in the host immune system may increase the risk for infection. ${ }^{3}$

Periodontitis, on the other hand is an inflammatory disease process that results in permanent destruction of the supporting apparatus of the teeth, including the loss of gingival connective tissue, destruction of the periodontal ligament and resorption of alveolar bone. ${ }^{4}$ It is associated with increased prevalence of cardiovascular morbidity and mortality. There is a rationale that indicates that the presence of periodontal pockets which can harbor pathogenic microorganisms can evoke a host response and elicit a systemic effect. ${ }^{5}$

Inflammatory diseases induce the production of proinflammatory cytokines such as TNF- $\alpha$, IL- 1 , and IL-6. ${ }^{6,7}$ Secretion of TNF- $\alpha$ by adipose tissue triggered by lipopolysaccharides from periodontal Gram- negative bacteria promotes hepatic dyslipidemia and decreases insulin sensitivity. ${ }^{8,9}$

Adipose tissue releases proinflammatory cytokines and hormones referred to as adipocytokines, which induce inflammatory processes and oxidative stress disorders, generating a similar pathophysiology between the both diseases. These suggest a potential interaction among obesity, periodontitis, and chronic disease incidence. This overview discusses the association between obesity and periodontal disease. Articles for this overview were procured from search engines google, google scholar and pubmed.

\section{Etiological factors}

The various factors contributing to obesity are

1. Genetics

2. Metabolic factors

3. Diet

4. Physical inactivity

5. Medication

In obese patients there is increased resistance to insulin. This leads to

i. Hyperinsulinemia

To compensate the resistance in tissues, especially adipose, muscular and hepatic, there is increased production of insulin by beta cells relative to level of glucose. This excess insulin increases insulin resistance in tissues and worsens ii. Hyperglycemia

Since there is increased resistance to insulin, circulating glucose is not taken by the cells and leads to increased blood glucose levels

iii. Hyperlipidemia

Alteration in liver metabolism leads to increase in liver lipogenesis and lipolysis in adipocytes.

Human adipose tissue is basically composed of

1. Brown adipose tissue which is responsible for thermogenic activity of tissue.

2. White adipose tissue which has fat storage function. It has different cell types namely fibroblasts, preadipocytes, mature adipocytes and macrophages. 


\section{Pathophysiology of obesity}

Normally, adipose tissue is populated by $5-10 \%$ macrophages. In obesity there is $60 \%$ macrophage infiltration, which keeps the body in a proinflammatory state. Also, there is release of fasting whole body free fatty acids and glycerol from adipocytes which promote insulin resistance. Until recently adipose tissue was believed to be inert tissue that stored fat. Adipose tissue is a metabolically active endocrine gland which secretes adipokines.

Insulin resistance occurs due to increased levels of TNF Alpha levels which inhibits insulin induced phosphorylation of insulin receptor substrate -1 and blocks translocation of glucose transporting protein (GLUT-4) to the cell membrane.

Increased free fatty acid release occurs due to alteration in perilipin expression. Perilipins are phosphoproteins on adipocytes that act as gatekeepers and prevent lipase to release FFA by hydrolyzingtriacylglycerol.

In obesity there is deficiency of perilipins which leads to increased release of excess free fatty acids.

\section{Obesity and inflammation}

Adipose tissue secretes immunomodulatory factors which stimulate adipose cells to secrete adipokines. Adipokines are key players in obesity and periodontal disease. The adipokines can be divided into pro and anti-inflammatory adipokines. The proinflammatory adipokines include:

i. Leptin

ii. Resistin

iii. Visfatin

iv. Lipocalin

v. TNF - Alpha

vi. PAI- 1

vii. Tissue factor

viii. Monocyte chemotactic protein -1

ix. TGF beta -1

x. iNO synthase

The anti-inflammatory adipokines include:

I. Leptin

II. Adipokines

III. IL-6

\section{Leptin}

Leptin is the first discovered adipokine in 1994 and is a pleiotropic cytokine. It is a $16 \mathrm{KDa}$ nonglycosylated peptide hormone which regulates weight control, insulin sensitivity, inflammation and controls appetite. It acts on hypothalamus and controls the satiety signal. It is similar in action to interleukin 6 and 11. It has a dual action i.e. pro and antiinflammatory action. The anti-inflammatory action is by stimulation of monocyte and macrophage function. In obesity, the levels of leptin are known to increase and in fasting, its level decreases.

It stimulates bone formation by increasing the life span of osteoblasts. On the other hand, it has a suppressive effect on bone formation. It acts as a lipostat that regulates adipose tissue mass. Faroogi et $\mathrm{al}^{10}$ in an animal study conducted in mice found that mutations in $\mathrm{OB}$ gene encoding leptin (causing leptin deficiency) lead to hyperphagia and severe obesity in mice. In obesity, the levels of leptin are known to increase since there is resistance to leptin at receptor level.

\section{Visfatin}

It is the most recently identified adipocytokine produced by visceral adipose tissue. It has insulin like action. It acts as a growth factor for early beta cells. In obesity, the levels of visfatin are increased to maintain euglycemia. Nogueira in 2013, found out that oral micro-organisms increase the levels of visfatin and thus proved that it plays a role in periodontal disease. ${ }^{11}$

Pradeep et al in 2011, 2012 found out that periodontal disease in obese individual correlated with GCF visfatin levels. ${ }^{12,13}$

\section{Lipocalin}

It is an adipokine which has bacteriostatic effects. Lipocalin 2 also known as neutrophil gelatinase-associated lipocalin, siderocalin, and $24 \mathrm{p} 3$ is a member of a large superfamily of proteins that includes RBP4. Lipocalins are small generally secreted proteins with a hydrophobic ligand binding pocket.

\section{Adiponectin}

Is a $30 \mathrm{KD}$ polypeptide which increases insulin sensitivity. It also decreases the levels of circulating fatty acids, triglycerides in liver and muscle. The levels are known to be decreased in obese humans and type II diabetes patients. It has increased osteoblastic action. It accounts for $0.05 \%$ of total serum proteins. It is involved in both glucose and lipid metabolism. It has anti-atherogenic and anti-inflammatory property.

\section{Resistin}

Belongs to RELM. Resistin originates from inflammatory cells in fatty tissues. During inflammation, it acts on adipocytes and causes insulin resistance. The levels of resistin are known to increase with increase in obesity. It has been known to form a link between diabetes and periodontitis. Rejala et al in 2002 found out that resistin causes resistance to insulin, thus the name. ${ }^{14}$ Kawanami et al, 2004 found out that human resistin was related to inflammatory process rather than insulin resistance. ${ }^{15}$ Snophia et al in 2017 found out a positive association between periodontal disease and GCF resistin levels. ${ }^{16}$

\section{TNF alpha}

It increases adipocyte lipolysis and also increases insulin resistance in peripheral tissues. It is secreted by macrophages. It is a known inhibitor of adiponectin. Since it is an inhibitor of adiponectin, there is an interesting fact that if the levels of TNF alpha are increased to 10-fold in young obese individuals, there is chance of periodontal disease in later life of the person even if the BMI is back to normal. It enhances periodontal degradation. TNF alpha in GCF has a positive association with BMI in subjects without periodontal disease. It is known to promote hepatic dyslipidaemia and decreases insulin level. 


\section{PAI- 1(Plaminogen - activator inhibitor)}

It causes agglutination of blood. It increases gingival inflammation and risk of ischemic vascular disease. It decreases blood flow in periodontium of obese patients and promotes periodontitis.

\section{Interleukin - 6}

Increased in obese adipose tissue. 10-fold increase than lean individuals.Increases lipolysis and fat oxidation. IL-6 expression is higher in visceral adipocytes than in peripheral adipocytes. More than $90 \%$ of IL- 6 is produced by cells other than adipocytes.

\section{Chemerin / TIG-2/ RARRES-2}

It has its origin from platelets and adipocytes. It circulates in blood as pro-chemerin; gets converted to chemerin in the presence of enzyme ACE (Angiotensin converting enzyme). It functions as chemokine and induces leukocyte migration. It acts as a bridge between innate and adaptive immunity via chemotaxis of macrophages. It has a role in obesity.

\section{Apelin}

Apelin is another short peptide released from adipocytes upon stimulation. Apelin exists in at least three forms, consisting of 13,17 , or 36 amino acids, all originating from a common 77-amino-acid precursor. Apelin synthesis is stimulated by insulin and plasma apelin levels increased in obesity associated insulin resistance and hyperglycemia.It is a short peptide produced from adipocytes upon stimulation.

\section{Angiotensinogen}

It has a vasoconstrictive effect on blood vessels.

\section{Vasculo-endothelial growth factor}

It contributes to artheromatous changes.

\section{Omentin / interlectin}

It has its origin from stromal- visceral vascular cells. It is a $33 \mathrm{KD}$ protein found in intenstinalpaneth cells.

\section{C-reactive protein}

The levels are known to increase in obese individuals. It is a risk factor for development of cardio-vascular disease and type -II diabetes mellitus.

\section{Effect of obesity on bone metabolism}

Obesity leads to overproduction of IL-1, 6 and TNF - alpha which leads to increased activity of osteoclasts by regulating RANK/RANKL/OPG.

\section{Effect of periodontal pathogens on obesity}

Goodson et al in 2009 proposed 3 mechanisms by which periodontal pathogens have an effect on obesity. ${ }^{17}$ Infectobesity proponent(Increased metabolic efficiency), Increase weight gain by increasing appetite and Redirect energy metabolism by facilitating insulin resistance. This happens by increasing the levels of TNF - alpha, which lead to increased insulin resistance. Also, he proposed that even a small excess in calorie consumption with no change in diet / exercise can lead to obesity

\section{Obesity and periodontitis}

Obesity is considered to be the second strongest risk factor for periodontal destruction. ${ }^{18}$ The first report on the relationship between obesity and periodontal disease appeared in 1977, when Perlstein et al. observed histopathologic changes in the periodontium in hereditary obese Zucker rats. ${ }^{19}$ Using ligature-induced periodontitis, they found alveolar bone resorption to be greater in obese animals compared with non-obese rats. Also, it seemed that under healthy oral conditions, obesity per se does not promote pathologic periodontal alterations; however, in response to bacterial plaque accumulation, periodontal inflammation and destruction were more severe in obese animals. Later on, the hypothesis of obesity as a risk factor for periodontal disease was supported by epidemiological studies.

In 1998, Saito et al. analyzed 241 healthy Japanese individuals and showed, for the first time, an association between obesity and periodontal disease in humans. In addition, studies have indicated that the fat distribution pattern plays a crucial role in the association with periodontitis. ${ }^{20}$

Another recent study by Saito et al. concluded that obesity is associated with deep periodontal pockets, independent of glucose tolerance status.

Genco et al. analyzed National Health and Nutrition Examination Survey (NHANES III) data and demonstrated that BMI was positively correlated with the severity of periodontal attachment loss; they found that this relationship is modulated by insulin resistance. ${ }^{21}$

The underlying biological mechanisms for the association of obesity with periodontitis are not well known; however, adipose-tissue-derived cytokines and hormones may play a key role. Fat tissue is not merely a passive triglyceride reservoir of the body, but also produces a vast amount of cytokines and hormones, collectively called adipokines or adipocytokines, which in turn may modulate periodontitis.

\section{Effect of obesity on host immunity}

Obesity leads to decreased $T$ cell mediated immune response and decreased lymphocyte function and NK cell action. Also, it alters chemotaxis and phagocytosis of PMN and macrophages.

1. Impaired tissue healing

\section{Obesity and ROS (Reactive Oxygen Species)}

ROS are products of normal cellular metabolism. Since obese individuals are at a pro-inflammatory state, there is excess production of ROS in them. This overproduction of ROS induces damage by oxidising DNA, lipids and proteins. This is the mechanism behind gingival oxidative damage and progression of Periodontitis. 


\section{Genetics and obesity}

Factors related to genetics and obesity include: Gene expression, Altered micro RNA expression, Posttranscriptional modulation of cytokine mRNA, Increased periodontal inflammation, Genome wide association studies and Specific mutations in key genes.

\section{Obesity and mortality}

Contradictory results

1. Increased mortality at higher or lower BMI

2. World Health Organization Global Observatory states that at least 2.8 million people worldwide die each year as a result of overweight or obesity.

Focussed on 4 main themes of association which include: Overweight/obesity and periodontitis prevalence, Incidence, Response to periodontal therapy and periodontal disease biomarkers

Based on A Measurement Tool to Assess systematic Reviews (AMSTAR) for the bias assessment of systematic reviews, the majority of the included reviews were deemed to be of moderate-to-high quality

In a study done by Elangovan et al., in 2014, the impact of obesity on peri- implant inflammation was assessed.Statistically significant correlation between interleukin-1beta in peri-implant sulcular fluid and waist circumference in 73 periodontal maintenance patients with implants. $^{22}$

Heterogenecity of the studies

1. Different sample size

2. Preliminary calibration

3. Confounder variables

4. Age range

5. Examiners not calibrated

6. To achieve homogenecity

7. Working with paired samples

8. Avoid confounding

9. Multicentre studies needed

Evidence from systematic reviews and meta-analysis

Benjamin W Chaffee et al conducted a systematic review in 2010 to find out the association between obesity and periodontal disease. He included 554 unique citations; among all the studies 70 studies met inclusion criteria. 41 studies showed positive association and the odds ratio was 1.35. The association was stronger in younger adults, women and non-smokers. There was increased prevalence of PD with increasing BMI. However, since the temporality cannot be proved, there is limited evidence of either obesity being a risk factor for periodontal disease or that periodontitis increases risk of weight gain. ${ }^{23}$

\section{Conclusion}

Increasing evidence of obesity as a risk indicator for periodontitis is merely a beginning and does not yet facilitate our understanding of the possible impact for patient care. Although this relationship needs further investigation, periodontists should counsel obese persons regarding the possible oral complications of obesity, to diminish morbidity for these individuals. This includes the measurement of body mass index and waist circumference for periodontal risk assessment on a regular basis.

\section{Source of funding}

None.

\section{Conflict of interest}

None.

\section{References}

1. Obesity and Overweight. World Health Organization; 2017.

2. Ling Yang, Ping Li, Suneng Fu, Ediz S. Calay, Go khan S. Hotamisligil. Defective Hepatic Autophagy in Obesity Promotes ER Stress and Causes Insulin resistance. Cell Metab 2010; 11:467-78.

3. Dalla Vecchia CF, Susin C, Rösing CK, Oppermann RV, Albandar JM. Overweight and obesity as risk indicators for Periodontitis in Adults. J Periodontol 2005;76:1721- 8.

4. Nield-Gehrig JS, Willmann DE. Foundations of periodontics for the dental hygienist (Lippincott Williams \& Wilkins, 2007).

5. Al-Zahrani MS, Bissada NF, Borawskit EA. Obesity and periodontal disease in young, middle-aged and older adults. $J$ Periodontol 2003;74:610-5.

6. Beck J, Garcia R, Heiss G, Vokonas P, Offenbacher S Periodontal disease and cardiovascular disease. J Periodontol 1996;67(10):1123-37.

7. Loos BG. Systemic markers of inflammation in periodontitis. $J$ Periodontol 2005;76(11):2106-15.

8. Saito T, Shimazaki Y, Koga T, Tsuzuki M, Oshima A. Relationship between upper body obesity and periodontitis. $J$ Dent Res 2001;80:1631-6.

9. Nishimura F, Iwamoto Y, Mineshiba J, Shimizu A, Soga Y, Murayama Y. Periodontal disease and diabetes mellitus: the role of tumor necrosis factor-alpha in a 2-way relationship. $J$ Periodontol 2003;74:97-102.

10. Farooqi IS, Matarese G, Lord GM, Keogh JM, Lawrence E, Agwu C, et al. Beneficial effects of leptin on obesity, T cell hyporesponsiveness, and neuroendocrine/metabolic dysfunction of human congenital leptin deficiency. J Clin Investig 2002;110(8):1093-103.

11. Nogueira AV, Nokhbehsaim M, Eick S, Bourauel C, Jäger A, Jepsen $\mathrm{S}$ et al. Regulation of visfatin by microbial and biomechanical signals in PDL cells. Clin Oral Investig 2014;18(1):171-8.

12. Pradeep AR, Raghavendra NM, Prasad MR, Kathariya R, Patel SP, Sharma A. Gingival crevicular fluid and serum visfatin concentration: their relationship in periodontal health and disease. J Periodontol 2011;82(9):1314-9.

13. Raghavendra NM, Pradeep AR, Kathariya R, Sharma A, Rao NS, Naik SB. Effect of non surgical periodontal therapy on gingival crevicular fluid and serum visfatin concentration in periodontal health and disease. Dis Markers 2012;32(6):383-8.

14. Gabriely I, MA XH, Yang XM, Atzmon G, Rajala MW, Berg $\mathrm{AH}$ et al. Removal of visceral fat prevents insulin resistance and glucose intolerance of aging: an adipokine-mediated process? Diabetes 2002;51:2951-8.

15. Kawanami D, Maemura K, Takeda N, Harada T, Nojiri T, Imai $\mathrm{Y}$, et al. Direct reciprocal effects of resistin and adiponectin on vascular endothelial cells: a new insight into adipocytokineendothelial cell interactions. Biochem Biophys Res Commun 2004;314(2):415-9.

16. Suresh S, Mahendra J, Singh G, Pradeep Kumar AR, Thilagar S, Rao N. Effect of nonsurgical periodontal therapy on plasmareactive oxygen metabolite and gingival crevicular fluid 
resistin and serum resistin levels in obese and normal weight individuals with chronic periodontitis. J Indian Soc

Periodontol 2018;22(4):310-6.

17. Goodson JM, Groppo D, Halem S, Carpino E. Is obesity an oral bacterial disease?. J Dent Res 2009;88(6):519-23.

18. Jagannathachary S, Kamaraj D. Obesity and periodontal disease. J Indian Soc Periodontol 2010;14(2):96.

19. Perlstein MI, Bissada NF. Influence of obesity and hypertension on the severity of periodontitis in rats. Oral Surg, Oral Med, Oral Pathol 1977;43(5):707-19.

20. Saito T, Shimazaki Y, Sakamoto M. Obesity and periodontitis. New Engl J Med 1998;339(7):482-3.

21. Genco RJ, Grossi SG, Ho A, Nishimura F, Murayama Y. A proposed model linking inflammation to obesity, diabetes, and periodontal infections. J Periodontol 2005;76:2075-84.
22. Elangovan S, Brogden KA, Dawson DV, Blanchette D, PaganRivera K, Stanford CM, et al. Body fat indices and biomarkers of inflammation: a cross-sectional study with implications for obesity and peri-implant oral health. Int J Oral Maxillofac Implants 2014;29(6).

23. Chaffee BW, Weston SJ. Association between chronic periodontal disease and obesity: a systematic review and meta-analysis. J Periodontol 2010;81(12):1708-24.

How to cite: Vijayalakshmi R, Thamaraiselvan, Mahendra J, Srinivasan S, Kumari BN. Obesity and periodontal disease An overview. IP Int J Periodontol Implantol 2020;5(1):1-5. 